\title{
On Classes of Stieltjes Type Operator-Valued Functions with Gaps
}

\author{
V. E. TSEKANOVSKII
}

\begin{abstract}
We introduce and investigate classes of operator-valued functions with gaps. which can be realized as fractional linear transformations of operator-valued transfer functions of conservative scattering systems.
\end{abstract}

Key words: Operator-valued functions, accretive extensions, conservative systems

AMS subject classification: 47 B 44

Classes of Stieltjes type operator-valued functions with gaps on the positive semi-axis (i.e., with intervals of holomorphy and definiteness) are considered. We prove criteria that a given function, whose values are operators in a finite-dimensional Hilbert space, belongs to these classes. Moreover, we investigate classes of Stieltjes type operator-valued functions which admit a realization, i.e., which can be represented as fractional linear transformations of operator-valued transfer functions of conservative scattering systems of the form

$$
\Theta=\left(\mathfrak{J}_{+} \subset \mathfrak{J} \subset \mathfrak{J}_{-}, A l, K, I, E\right)
$$

where $\left.A \mid \in\left[\mathfrak{S}_{+}, \mathfrak{S}_{-}\right], \operatorname{Im} A\right]=K K^{*}, A l \supset T \supset A, A^{*} \supset T^{*} \supset A, A$ is a closed Hermitian operator in $\mathfrak{S}$, and $T$ is closed with dense domain of definition in $\mathbf{S}$.

In the class of realizable Stieltjes type operator-valued functions the following subclasses are investigated:

1. the subclass, where $\overline{\mathfrak{D}(A)}=\mathfrak{S}, \mathfrak{D}(T) \neq \mathfrak{D}\left(T^{*}\right)$

2. the subclass, where $\overline{\mathfrak{D}(A)} \neq \mathfrak{S}, \mathfrak{D}(T) \neq \mathfrak{D}\left(T^{*}\right)$

3. the subclass, where $\overline{\mathfrak{D}(A)} \neq \mathfrak{S}, \mathfrak{D}(T)=\mathfrak{D}\left(T^{*}\right)$.

We prove analytical criteria for a given operator-valued function to belong to the mentioned subclasses (with gaps). These criteria are analoga, supplements, and refinements of some of the results stated by M.G. Krein and A.A. Nudel'man [7].

\section{$\S 1$ The classes $S_{ \pm}\left[\bigcup_{j=1}^{m}\left(\alpha_{j}, \beta_{j}\right)\right]$ of operator-valued functions}

According to M.G. Krein [8], a function $V(z)$, whose values are operators in a finite-dimensional Hilbert space $E$, will be called a Stieltjes type operator-valued function if the following conditions hold:

1. $V(z)$ is holomorphic on $\operatorname{Ext}[0, \infty):=\{z: z,[0, \infty)\}$

2. $V(z) \geq 0$ for $z<0$

3. $V(z)$ is an operator-valued R-function, i.e., $\operatorname{Im} V(z) / \operatorname{Im} z \geq 0$.

The class of Stieltjes type operator-valued functions will be denoted by $S$.

Let $\left\{\left(\alpha_{j}, \beta_{j}\right)\right\}_{j=1}^{m}$ be a system of mutually disjoint intervals on the positive semi-axis. 
Definition: By $S_{ \pm}\left[\bigcup_{j=1}^{m}\left(\alpha_{j}, \beta_{j}\right)\right]$ we denote the class of functions $V(z)$, whose values are operators in a finite-dimensional Hilbert space $E$, such that the following two conditions hold:

1. $V(z) \in S$.

2. $V(z)$ is holomorphic and positive on all intervals $\left(\alpha_{j}, \beta_{j}\right)$, i.e., $(V(z) f, f)>0$ for all $f \in E$, $f \neq 0$, and all $z \in\left(\alpha_{j}, \beta_{j}\right)\left(V(z)\right.$ is holomorphic and negative on the intervals $\left(\alpha_{j}, \beta_{j}\right)$, respectively, i.e., $(V(z) f, f)<0$ for all $f \in E, f \neq 0$, and all $\left.z \in\left(\alpha_{j}, \beta_{j}\right)\right)$.

Theorem 1: A scalar function $V(z)$ belongs to the classes $S_{ \pm}\left[U_{j=1}^{m}\left(\alpha_{j}, \beta_{j}\right)\right]$ if and only if the following two conditions hold:

(i) $V(z) \in S$.

(ii) $\prod_{j=1}^{m} \frac{\beta_{j}-z}{\alpha_{j}-z} V(z) \in S\left(\prod_{j=1}^{m} \frac{\alpha_{j}-z}{\beta_{j}-z} V(z) \in S\right.$, respectively).

Proof: First we consider the class $S_{+}\left[\bigcup_{j=1}^{m}\left(\alpha_{j}, \beta_{j}\right)\right]$. Let (i) and (ii) be fulfilled. Since (i), a well known theorem (see [7]) gives us

$$
V(z)=c \exp \int_{-\infty}^{+\infty}\left(\frac{1}{t-z}-\frac{t}{1+t^{2}}\right) f(t) d t
$$

where $c>0, f(t)$ is a summable function such thast $0 \leq f(t) \leq 1$ a.e. and $\int_{-\infty}^{+\infty}\left(1+t^{2}\right)^{-1} f(t) d t$ $<\infty$. Moreover, the representation (2) is unique. It is not hard to see that

$$
\frac{\beta_{j}-z}{\alpha_{j}-z}=c_{j} \exp \int_{\alpha_{j}}^{\beta_{j}}\left(\frac{1}{t-z}-\frac{t}{1+t^{2}}\right) f(t) d t .
$$

Since (ii), we get.

$$
\prod_{j=1}^{m} \frac{\beta_{j}-z}{\alpha_{j}-z} V(z)=c_{1} \exp \int_{-\infty}^{+\infty}\left(\frac{1}{t-z}-\frac{t}{1+t^{2}}\right) f_{1}(t) d t
$$

in an analogous way, where $c_{1}>0$ and the function $f_{1}(t)$ has the same properties as $f(t)$. Using (3) and (4), we obtain

$$
V(z)=c_{2} \exp \int_{-\infty}^{+\infty}\left(\frac{1}{t-z}-\frac{t}{1+t^{2}}\right) f_{2}(t) d t
$$

where

$$
f_{2}(t)=\left\{\begin{array}{l}
f_{1}(t) \quad \text { for } t \in \mathbb{R} \backslash \bigcup_{j=1}^{m}\left(\alpha_{j}, \beta_{j}\right) \\
f_{1}(t)-1 \text { for } t \in \bigcup_{j=1}^{m}\left(\alpha_{j}, \beta_{j}\right)
\end{array} .\right.
$$

Because of the uniqueness of the representation (2) it follows

$$
V(z)=c \quad \exp \int_{\mathbb{R} \backslash \bigcup_{j=1}^{m}\left(\alpha_{j}, \beta_{j}\right)}\left(\frac{1}{t-z}-\frac{t}{1+t^{2}}\right) f(t) d t,
$$

where $c>0,0 \leq f(t) \leq 1$ a.e. on $\mathbb{R} \backslash \bigcup_{j=1}^{m}\left(\alpha_{j}, \beta_{j}\right)$. By a well known theorem (see [7]), the relation (5) implies that $V(z)$ is holomorphic and positive on all intervals $\left(\alpha_{j}, \beta_{j}\right)$.

Now assume that $V(z) \in S_{+}\left[\bigcup_{j=1}^{m}\left(\alpha_{j}, \beta_{j}\right)\right]$. Then $V(z) \in S_{+}\left[\left(\alpha_{1}, \beta_{1}\right)\right]$. We will show that the inclusion $\left(\left(\beta_{1}-z\right) /\left(\alpha_{1}-z\right)\right) V(z) \in S$ is true. In fact, setting $\xi=\left(\beta_{1}-z\right) /\left(\alpha_{1}-z\right)$ and $V(\xi)=V(z)$ 
we get

$$
\frac{\operatorname{Im} V_{1}(\xi)}{\operatorname{Im} \xi}=\frac{\left|\alpha_{1}-z\right|^{2}}{\beta_{1}-\alpha_{1}} \frac{\operatorname{Im} V(z)}{\operatorname{Im} z} \geq 0,
$$

hence, $V_{1}(\xi) \in R$, i.e., $V_{1}(\xi)$ is an $R$-function. It is not hard to see that $z \in\left(\alpha_{1}, \beta_{1}\right)$ implies $\xi \in(-\infty, 0)$, and since $V(z) \in S_{+}\left[\left(\alpha_{1}, \beta_{1}\right)\right]$, it follows $V_{1}(\xi) \in S$. Now a theorem of M.G. Krein (see [8]) gives us $\xi V_{1}(\xi) \in R$. Thus $\left(\left(\beta_{1}-z\right) /\left(\alpha_{1}-z\right)\right) V(z) \in R$. Since $\left(\left(\beta_{1}-z\right) /\left(\alpha_{1}-z\right)\right) V(z) \geq 0$ if $z \in(-\infty, 0)$, we obtain $\left(\left(\beta_{1}-z\right) /\left(\alpha_{1}-z\right)\right) V(z) \in S$.

We will show that the implication

$$
\prod_{j=1}^{k} \frac{\beta_{j}-z}{\alpha_{j}-z} V(z) \in S(1<k<m) \Rightarrow \prod_{j=1}^{k+1} \frac{\beta_{j}-z}{\alpha_{j}-z} V(z) \in S
$$

is true. In fact, it is not hard to see that

$$
\prod_{j=1}^{k} \frac{\beta_{j}-z}{\alpha_{j}-2} V(z) \in S_{+}\left[\left(\alpha_{k+1}, \beta_{k+1}\right)\right] .
$$

Now by analogous arguments as above we get

$$
\frac{\beta_{k+1}-z}{\alpha_{k+1}-z} \prod_{j=1}^{k} \frac{\beta_{j}-z}{\alpha_{j}-z} V(z)=\prod_{j=1}^{k+1} \frac{\beta_{j}-z}{\alpha_{j}-z} V(z) \in S .
$$

Thus the first part of the theorem is proved.

Now let $V(z) \in S_{-}\left[\bigcup_{j=1}^{m}\left(\alpha_{j}, \beta_{j}\right)\right]$. We will show that the ()-part of (ii) is true. It is not hard to see (cf. [6]) that $V(z) \in R$ if and only if $-V(z)^{-1} \in R$. Thus, the relation $V(z) \in S_{-}\left[\left(\alpha_{1}, \beta_{1}\right)\right]$ implies $-V(z)^{-1} \in R$ and $-V(z)^{-1}>0, z \in\left(\alpha_{1}, \beta_{1}\right)$. Setting $\xi=\left(\beta_{1}-z\right) /\left(\alpha_{1}-z\right)$ and $V(\xi)=-V(z)^{-1}$, we get

$$
\frac{\beta_{1}-z}{\alpha_{1}-z}\left(-\frac{1}{V(z)}\right) \in R, \text { hence }-\left(\frac{\beta_{1}-z}{\alpha_{1}-z}\left(-\frac{1}{V(z)}\right)\right)^{-1}=\frac{\alpha_{1}-z}{\beta_{1}-z} V(z) \in R .
$$

Since $\frac{\alpha_{1}-z}{\beta_{1}-z} V(z) \geq 0$ if $z \in(-\infty, 0)$, it follows $\frac{\alpha_{1}-z}{\beta_{1}-z} V(z) \in S$. Using an analogous induction method as in the first part of the proof we obtain the ( )-part of (ii).

Now assume (i) and (ii) $/()$-part. Consider the function $-V(z)^{-1} \in R$ and use analogous arguments as in the proof of the sufficiency in the first part. This gives us that $-V(z)^{-1}$ is holomorphic and positive on all intervals $\left(\alpha_{j}, \beta_{j}\right)$. Thus the theorem is proved

Theorem 2: A function $V(z)$, whose values are operators in a finite-dimensional Hilbert space $E$, belongs to the classes $S_{ \pm}\left[\bigcup_{j=1}^{m}\left(\alpha_{j}, \beta_{j}\right)\right]$ if and only if the following two conditions hold:

(i) $V(z) \in S$.

(ii) $\prod_{j=1}^{m} \frac{\beta_{j}-z}{\alpha_{j}-z} V(z) \in S\left(\prod_{j=1}^{m} \frac{\alpha_{j}-z}{\beta_{j}-z} V(z) \in S\right.$, respectively).

Proof: Let $V(z) \in S_{+}\left[\bigcup_{j=1}^{m}\left(\alpha_{j}, \beta_{j}\right)\right]$. Considering the scalar function $\left.(Y z) f, f\right) \in S$ and using Theorem 1 we get

$$
\prod_{j=1}^{m} \frac{\beta_{j}-z}{\alpha_{j}-z}(V(z) f, f)=\left(\prod_{j=1}^{m} \frac{\beta_{j}-z}{\alpha_{j}-z} V(z) f, f\right) \in S .
$$

Hence, the operator-valued function $\prod_{j=1}^{m} \frac{\beta_{j}-z}{\alpha_{j}-z} V(z)$ belongs to $S$. 
The sufficiency of conditions (i) and (ii) is trivial. The proof for the class $S_{-}\left[\bigcup_{j=1}^{m}\left(\alpha_{j}, \beta_{j}\right)\right]$ is analogous. Thus the theorem is proved

Definition: We will say that a function $V(z)$, whose values are operators in a finite-dimensional Hilbert space $E$, belongs to the class

$$
S_{+}\left[\bigcup_{j=1}^{m}\left(\alpha_{j}, \beta_{j}\right) \cap S_{-}\left[\bigcup_{j=1}^{n}\left(c_{j}, d_{j}\right)\right]\right.
$$

if the following three conditions hold:

1. $V(z) \in S$.

2. $V(z)$ is holomorphic and positive on the intervals $\left(\alpha_{j}, \beta_{j}\right)(j=1, \ldots, m)$.

3. $V(z)$ is holomorphic and negative on the intervals $\left(c_{k}, d_{k}\right)(k=1, \ldots, n)$.

Theorem 2 immediately implies the following

Theorem 3: A function $V(z)$, whose values are operators in a finite-dimensional Hilbert space $E$, belongs to the class $S_{+}\left[\bigcup_{j=1}^{m}\left(\alpha_{j}, \beta_{j}\right)\right] \cap S_{-}\left[\bigcup_{k=1}^{n}\left(c_{k}, d_{k}\right)\right]$ if and only if the following two conditions hold:
1. $V(z) \in S$.
2. $\prod_{j=1}^{m} \frac{\beta_{j}-z}{\alpha_{j}-z} \prod_{k=1}^{n} \frac{c_{k}-z}{d_{k}-z} V(z) \in S$.

\section{$\$ 2$ Realizable operator-valued functions of the class $S_{ \pm}\left[U_{j=1}^{m}\left(\alpha_{j}, \beta_{j}\right)\right]$}

Let $A$ be a closed Hermitian operator in a Hilbert space $\mathfrak{S}$, whose defect numbers are finite and coincide. This operator can be considered as acting from $\mathfrak{J}_{0}=\overline{\mathfrak{D}(A)}$ into $\mathfrak{W}$. Let $A^{*}$ be the adjoint operator. Clearly, $\overline{\mathfrak{D}\left(A^{*}\right)}=\mathfrak{S}$ (where the closure is taken in $\mathfrak{S}$ ). We set $\mathfrak{S}_{+}=\mathfrak{D}\left(A^{*}\right)$ and introduce the scalar product $(f, g)_{+}=(f, g)+\left(A^{*} f, A^{*} g\right)\left(f, g \in S_{+}\right)$. We consider the rigged Hilbert space $\mathfrak{J}_{+} \subset \mathfrak{S}_{\mathbf{Z}} \subset \mathfrak{S}_{-}$(cf. [2]).

We will say that a closed and densely defined operator $T$ in $\mathfrak{g}$ belongs to the class $\Omega_{\mathcal{A}}$ if the following two conditions

1. $T \supset A, T^{\bullet} \supset A(A$ is closed and Hermitian)

2. $-i$ is a regular point of $T$

are fulfilled.

A bounded operator $A l: \mathfrak{S}_{+} \rightarrow \mathfrak{S}_{-}$(i.e., $A \mid \in\left[\mathfrak{X}_{+}, \mathfrak{S}_{-}\right]$) is called a biextension of the Hermitian operator $A$ if $A l \supset A$ and $\left.A\right|^{*} \supset A$. Identifying the dual space of $\mathfrak{S}_{ \pm}$with $\mathfrak{S}_{\mp}$, we see that $A)^{*} \in\left[\mathfrak{H}_{+}, \mathfrak{S}_{-}\right]$. If $A l=A l^{*}$, then $A l$ is called a selfadjoint biextension of $A$.

By $\hat{A}$ we denote the restriction of $A \mid$ to $\mathfrak{D}(\hat{A})=\left\{f \in \mathfrak{S}_{+}: A \mid f \in \mathfrak{S}\right\}$. It is called a quasikernel of $A \mathrm{l}$ (cf. $[10,11]$ ). A selfadjoint biextension is called a strong biextension if $\hat{A}=\hat{A}^{-}$(cf. $\left.[10,11]\right)$.

Let $T \in \Omega_{A^{*}}$. Then $A l \in\left[\mathfrak{Y}_{+}, \mathfrak{S}_{-}\right]$is called a (*)-extension of $T$ if

$$
A l \supset T \supset A, A l^{\bullet} \supset T^{\bullet} \supset A \text {. }
$$

Moreover, if $A_{R}=\left(A|+A|^{*}\right) / 2$ is a strong selfadjoint biextension, then $A$ il is called a correct (*)-extension of $T$.

By definition, the class $\Lambda_{A}$ denotes the set of all operators $T \in \Omega_{A}$ such that $A$ coincides with the maximal common Hermitian part of $T$ and $T^{*}$. 
Definition: The operator colligation

$\boldsymbol{\Theta}=\left(\begin{array}{ccc}\mathfrak{S}_{+} \subset \mathfrak{S}_{\mathfrak{J}} \subset \mathfrak{S}_{-} & K & J \\ & & E\end{array}\right)$

is called rigged if the following four conditions hold:

1. $J=J^{*}=J^{-1}(\operatorname{dim} E<\infty)$.

2. $K$ is a bounded linear operator from $E$ into $\mathfrak{S}_{-}$.

3. $A l$ is a correct $(*)$-extension of $T \in \Lambda_{A}$, and

$\operatorname{Im} A l=\left(A|-A|^{*}\right) / 2 \mathrm{i}=K J^{*} K^{*}$.

4. The ranges of $K$ and Im $A$ l coinside.

The operaror-valued function

$W_{\Theta}(z)=I-2 \mathrm{i} K^{*}(A \mid-z I)^{-1} K J$

is called a Livsic type characteristic function of the colligation $\theta$.

Furthermore, we introduce the function

$V_{\Theta}(z)=K^{*}\left(\left.A\right|_{R}-z I\right)^{-1} K$.

It is well known (cf. $[3,9]$ ) that the functions $V_{\Theta}(z)$ and $W_{\Theta}(z)$ are associated by the relations

$$
V_{\Theta}(z)=\mathrm{i}\left(W_{\Theta}(z)+I\right)^{-1}\left(W_{\Theta}(z)-I\right) J \text { and } W_{\Theta}(z)=\left(I+\mathrm{i} V_{\Theta}(z) J\right)^{-1}\left(I-\mathrm{i} V_{\Theta}(z) J\right)
$$

We consider the conservative system (cf. [9])

$$
\begin{aligned}
& (A \mid-z I)_{X}=K J \varphi_{-} \\
& \varphi_{+}=\varphi_{-}-2 \mathrm{i} K^{*} x,
\end{aligned}
$$

where $x \in \mathfrak{W}_{+} \varphi_{ \pm} \in E, \varphi_{-}$is the so-called input vector, $\varphi_{+}$is the output vector, and $x$ is the inner state. It is not hard to see that the transfer function $\Pi(z)$ of such a system (i.e., $\varphi_{+}=\Pi(z) \varphi_{-}$) coincides with the operator function $W_{\Theta}(z)$. If $J \neq I$, then the system is called a crossing system and if $J=I$, it is called a scattering system (cf. [1]). In the following we will write a conservative system $\theta$ in the form of a rigged operator colligation.

Definition: A function $V(z)$, whose values are operators in a finite-dimensional Hilbert space $E$, is called realizable if it can be represented as

$$
V(z)=V_{\Theta}(z)=K^{*}\left(A_{R}-z I\right)^{-1} K=\mathrm{i}\left(W_{\Theta}(z)+I\right)^{-1}\left(W_{\Theta}(z)-I\right),
$$

where $\theta$ is a conservative scattering system of the form (7).

Theorem 4: Let $V(z)$ be a realizable function, whose values are operators in a finite-dimensional Hilbert space $E$, i.e., $V(z)=K^{\bullet}\left(A \|_{R}-z I\right)^{-1} K$. Let $A>0$ and let $(\alpha, \beta)$ be an arbitrary interval of the positive semi-axis. Then $V(z)$ belongs to the class $S_{+}[(\alpha, \beta)$ if and only if the following two conditions hold:

1. $\left.A\right|_{R} \geq 0$.

2. For an arbitrary set $\left\{z_{j}\right\}_{j=1}^{p}$ of non-real complex numbers such that $z_{j} \neq \overline{z_{1}}$ and for all $\varphi_{i} \in N_{z_{i}}$ (where $N_{z_{i}}$ is the deficiency space of $A$ ) it holds

$$
\sum_{i, 1=1}^{p}\left(B\left(z_{i}, z_{1}\right) \varphi_{i}, \varphi_{l}\right) \geq 0
$$


where

$$
B(\lambda, \mu) \doteq \frac{\beta-\alpha}{(\alpha-\lambda)(\alpha-\bar{\mu})} A_{R}+\frac{\alpha \beta-\beta(\lambda+\bar{\mu})+\lambda \bar{\mu}}{(\alpha-\lambda)(\alpha-\bar{\mu})} I .
$$

Proof: Assume that the conditions (14) and (15) hold. We will show that $V(z) \in S_{+}[(\alpha, \beta)]$. Since $V(z)$ is realizable, there exists a conservative scattering system

$$
\Theta=\left(\begin{array}{lll}
\mathfrak{S}_{+} \subset \mathfrak{S}_{\boldsymbol{Z}} \subset \mathfrak{S}_{-} & K & \boldsymbol{J}
\end{array}\right)
$$

such that $V(z)=V_{\Theta}(z)=K^{\bullet}\left(\left.A\right|_{R}-z I\right)^{-1} K$. The operator $A$ il a $(\bullet)$-extension of some densely defined closed operator $T$, i.e., condition (6) holds, where $A$ is the common maximal Hermitian part of $T$ and $T^{*}$. Let $N_{z}$ be the deficiency space of the Hermitian operator $A$ and let $\left\{z_{j}\right\}_{j=1}^{P}$ be an arbitrary set of non-real complex numbers such that $z_{i} \neq \bar{z}_{l}$. Moreover, let $\varphi_{i} \in N_{z_{i}}$. According to [11], there exists a vector $h_{i} \in E$ such that

$$
\varphi_{i}=\left(\left.A\right|_{R}-z I\right)^{-1} K h_{j} \quad(i=1, \ldots, p) .
$$

Set $w_{i}=\left(\beta-z_{i}\right) /\left(\alpha-z_{j}\right)$. We will prove the inequality

$$
\sum_{i, l=1}^{p}\left(\frac{w_{i} V\left(z_{i}\right)-\overline{w_{1}} V\left(\overline{z_{l}}\right)}{z_{i}-\overline{z_{l}}} h_{i}, h_{l}\right) \geq 0 \text {. }
$$

In fact,

$$
\begin{aligned}
& \sum_{i, l=1}^{p}\left(\frac{w_{i} V\left(z_{i}\right)-\overline{w_{l}} V\left(\overline{z_{1}}\right)}{z_{i}-\overline{z_{I}}} h_{i}, h_{1}\right) \\
& =\sum_{i, I=1}^{P}\left(\frac{w_{i}\left(\left.A\right|_{R}-z_{i} I\right)^{-1}-\bar{w}_{l}\left(\left.A\right|_{R}-\bar{z}_{i} I\right)^{-1}}{z_{i}-z_{l}} K, K h_{1}\right) \\
& =\sum_{i, l=1}^{p}\left(\frac{\left(\left.A\right|_{R}-\bar{z}_{l} I\right)^{-1}\left(w_{i}\left(\left.A\right|_{R}-\bar{z}_{l} I\right)-\bar{w}_{l}\left(\left.A\right|_{R}-z_{i} I\right)\right)\left(\left.A\right|_{R}-z_{i} I\right)^{-1}}{z_{i}-\overline{z_{l}}} K h_{i}, K h_{l}\right) \\
& =\sum_{i, l=1}^{p}\left(\left(\frac{w_{i}-\bar{w}_{l}}{z_{i}-\bar{z}_{l}} A_{k}+\frac{z_{i} \bar{w}_{l}-\bar{z}_{l} w_{i}}{z_{j}-\bar{z}_{l}} I\right) \varphi_{i}, \varphi_{l}\right) \\
& =\sum_{i, l=1}^{p}\left(\left(\left.\frac{\beta-\alpha}{\left(\alpha-z_{i}\right)\left(\alpha-\overline{z_{l}}\right)} A\right|_{R}+\frac{\alpha \beta-\beta\left(z_{i}+\overline{z_{l}}\right)+z_{i} \overline{z_{l}}}{\left(\alpha-z_{j}\right)\left(\alpha-\overline{z_{l}}\right)} I\right) \varphi_{i}, \varphi_{l}\right) \\
& =\sum_{i, l=1}^{p}\left(B\left(z_{j}, z_{1}\right) \varphi_{j}, \varphi_{l}\right) \geq 0 \text {. }
\end{aligned}
$$

Setting $p=1, z_{1}=z, h_{1}=h$, we obtain from (17) and (18)

$$
\left(\frac{\frac{\beta-z}{\alpha-z} V(z)-\frac{\beta-\bar{z}}{\alpha-\bar{z}} V(\bar{z})}{z-\bar{z}} h, h\right) \geq 0 \text { and hence } \frac{\operatorname{Im}\left(\frac{\beta-z}{\alpha-z} V(z)\right)}{\operatorname{Im} z} \geq 0
$$

i.e., the operator-valued function $((\beta-z)(\alpha-z)) V(z)$ is an operator-valued $\mathrm{R}$-function. Now condition (14) implies that $V(z) \in S$ (cf. [4]). Since for $z<0$ we have $(\beta-z)(\alpha-z) \geq 0$ it holds $((\beta-z)(\alpha-z)) V(z) \in S$. By Theorem 2 we get $V(z) \in S_{+}[(\alpha, \beta)]$. 
Now assume $V(z) \in S_{+}[(\alpha, \beta)]$. Thus $V(z) \in S$ and hence $\left.A\right|_{R} z 0$ (cf. [4]). It remains to prove (15). In fact, by Theorem 2 the condition $V(z) \in S_{+}[(\alpha, \beta)]$ implies that $((\beta-z)(\alpha-z)) V(z) \in S$. Thus, the operator-valued function $((\beta-z)(\alpha-z)) V(z)$ has a representation of the form

$$
\frac{\beta-z}{\alpha-z} V(z)=\gamma+\int_{0}^{\infty} \frac{1}{t-z} d \sigma(t)
$$

where $\gamma \geq 0, \sigma(t)$ is a non-decreasing operator-valued function in $E$ such that $\int_{0}^{\infty}(1+t)^{-1} d \sigma(t)<\infty$. Let $\left\{z_{i}\right\}_{i=2}^{p}$ be an arbitrary set of non-real complex numbers such that $z_{i} \neq \bar{z}_{1}$. Let $\varphi_{j} \in N_{z_{i}}$. Then according to [11] there exist vectors $h_{i} \in E$ such that (17) holds. Setting $w_{i}=\left(\beta-z_{i}\right)\left(\alpha-z_{i}\right)$ we obtain

$$
\sum_{i, l=1}^{p}\left(\frac{w_{i} V\left(z_{i}\right)-\overline{w_{l}} V\left(\bar{z}_{1}\right)}{z_{i}-\overline{z_{l}}} h_{i}, h_{1}\right)=\sum_{i, J=1}^{p}\left(\int_{0}^{\infty} \frac{1}{\left(t-z_{i}\right)\left(t-\overline{z_{l}}\right)} d \sigma(t) h_{i}, h_{1}\right) \geq 0 .
$$

It is clear from the proof of the sufficiency part that the inequalities (15) and (18) are equivalent. Thus, the above inequality yields the needed result

Remark:If there is no gap, i.e., if $\alpha=\beta$, the inequality (15) holds trivially and we obtain the results of [4].

Theorem 5: Let $V(z)$ be a realizable function, whose values are operators in a finite-dimensional Hilbert space $E$, i.e., $V(z)=K^{*}\left(\left.A\right|_{R}-z I\right)^{-1} K$. Let $(\alpha, \beta)$ be an arbitrary interval of the positive semi-axis. Then $V(z)$ belongs to the class $S_{-}[(\alpha, \beta)]$ if and only if the following two conditions hold:

1. $A I_{R} \geq 0$.

2. For an arbitrary set $\left\{z_{i}\right\}_{i=1}^{p}$ of non-real complex numbers such that $z_{i} \neq z_{1}$ and for all $\varphi_{i} \in N_{z_{i}}$ (where $N_{z_{i}}$ is the deficiency space of $A$ ) it holds

$$
\sum_{i=1}^{p}\left(B\left(z_{i}, z_{1}\right) \varphi_{i}, \varphi_{1}\right) \geq 0,
$$

where

$$
B(\lambda, \mu)=\frac{\alpha-\beta}{(\beta-\lambda)(\beta-\bar{\mu})} A_{R}+\frac{\alpha \beta-\alpha(\lambda+\bar{\mu})+\lambda \bar{\mu}}{(\beta-\lambda)(\beta-\bar{\mu})} I .
$$

Proof: This theorem can be proved in the same way as Theorem $4\left(\operatorname{set} w_{j}=\left(\alpha-z_{j}\right)\left(\beta-z_{i}\right)\right)$

Note further that in the case $\alpha=\beta$ (i.e., if there are no gaps) Theorem 5 is an extension of results of [4]. Moreover, it is not hard to see that the above used method allows us to obtain analogous results for the classes

$$
S_{ \pm}\left[\bigcup_{j=1}^{m}\left(\alpha_{j}, \beta_{j}\right)\right] \text { and } S_{+}\left[\bigcup_{j=1}^{m}\left(\alpha_{j}, \beta_{j}\right)\right] \cap S_{-}\left[\bigcup_{j=1}^{n}\left(c_{j}, \alpha_{j}\right)\right] \text {. }
$$

In fact, we have the following

Theorem 6: Let $V(z)$ be a realizable function, whose values are operators in a finite-dimensional Hilbert space $E$, i.e., $V(z)=K^{*}\left(\left.A\right|_{R}-z I\right)^{-1} K$. Let $\left(\alpha_{j}, \beta_{j}\right)(j=1, \ldots, m)$ and $\left(c_{k}, d_{k}\right)$ $(k=1, \ldots, n)$ be arbitrary mutually disjoint intervals of the positive semi-axis. Then $V(z)$ belongs to the class $S_{+}\left[\bigcup_{j=1}^{m}\left(\alpha_{j}, \beta_{j}\right)\right] \cap S_{-}\left[\bigcup_{k=1}^{n}\left(c_{k}, d_{k}\right)\right]$ if and only if the following two conditions hold: 
1. $\left.A\right|_{R} \geq 0$.

2. For an arbitrary set $\left\{z_{i}\right\}_{i=1}^{P}$ of non-real complex numbers such that $z_{i} \neq \bar{z}_{1}$ and for all $\varphi_{i} \in N_{\mathbf{z}_{j}}$ (where $N_{\boldsymbol{z}_{\boldsymbol{i}}}$ is the deficiency space of $A$ ) it holds

$$
\sum_{i, l=1}^{p}\left(B\left(z_{i}, z_{1}\right) \varphi_{i}, \varphi_{1}\right) \geq 0
$$

where

$$
B(\lambda, \mu)=\left.\frac{w(\lambda)-w(\bar{\mu})}{\lambda-\bar{\mu}} A\right|_{R}+\frac{\lambda w(\bar{\mu})-\bar{\mu} w(\lambda)}{\lambda-\bar{\mu}}
$$

and

$$
w(\lambda)=\prod_{j=1}^{m} \frac{\beta_{j}-\lambda}{\alpha_{j}-\lambda} \prod_{k=1}^{n} \frac{c_{k}-\lambda}{d_{k}-\lambda}
$$

\section{\$3 Some subclasses of realizable Stieltjes type operator-valued functions with gaps}

By a result of M.G. Krein (see [8]) each Stieltjes type function $V(z)$, whose values are operators in a finite-dimensional Hilbert space $E$, can be represented in the form

$$
V(z)=\gamma+\int_{0}^{\infty} \frac{d \sigma(t)}{(t-z)}
$$

where $\gamma \geq 0, \sigma(t)$ is a non-decreasing operator-valued function in $E$ such that $\int_{0}^{\infty}(1+t)^{-1} d \sigma(t)<\infty$. According to [5], we introduce the following notion.

Definition: We will say that a Stieltjes type function $V(z)$, whose values are operators in a finite-dimensional Hilbert space $E$, belongs to the class $S(R)$, if $\gamma f=0$ for all $f$ of the subclass

$$
E_{\infty}^{\perp}=\left\{f \in E: \int_{0}^{\infty}(d \sigma(t) f, f)_{E}<\infty\right\} .
$$

As it was proved in [5], each operator-valued function $V(z) \in S(R)$ can be realized by a conservative scattering system $\Theta$, i.e., it holds (13).

Definition: Following [5], we introduce the following subclasses of $S(R)$ :

(i) The class $S^{\circ}(R)$ consisting of all $V(z) \in S(R)$ such that

$\int_{0}^{\infty}(d o(t) f, f)=\infty \quad(f \in E, f \neq 0)$.

(ii) The class $S^{1}(R)$ consisting of all $V(z) \in S(R)$ such that $\gamma=0$ and

$$
\int_{0}^{\infty}(d \sigma(t) f, f)<\infty \quad(f \in E)
$$

in the representation (23).

(iii) The class $S^{\circ \mathcal{L}}(R)$ consisting of all $V(z) \in S(R)$ such that $E_{\infty}^{\perp} \neq\{0\}$ and $E_{\infty}^{\perp} \neq E$. 
It is not hard to see that

$S(R)=S^{\circ}(R) \cup S^{1}(R) \cup S^{\circ}(R)$.

Definition: We introduce the following subclasses of $S(R), S^{\circ}(R), S^{1}(R)$ and $S^{01}(R)$.

(i) The class $S_{ \pm}\left[R, \cup_{j=1}^{m}\left(\alpha_{j}, \beta_{j}\right)\right]$ consisting of all $V(z) \in S(R)$ such that $V(z)$ is holomorphic and positive (negative) on all intervals $\left(\alpha_{j}, \beta_{j}\right)$.

(ii) The class $S_{ \pm}^{\circ}\left[R, \bigcup_{j=1}^{m}\left(\alpha_{j}, \beta_{j}\right)\right]$ consisting of all $V(z) \in S^{\circ}(R)$ such that $V(z)$ is holomorphic and positive (negative) on all intervals $\left(\alpha_{j}, \beta_{j}\right)$.

(iii) The class $S_{ \pm}^{1}\left[R, \cup_{j=1}^{m}\left(\alpha_{j}, \beta_{j}\right)\right]$ consisting of all $V(z) \in S^{1}(R)$ such that $V(z)$ is holomorphic and positive (negative) on all intervals $\left(\alpha_{j}, \beta_{j}\right)$.

(iv) The class $S_{ \pm}^{01}\left[R, \cup_{j=1}^{m}\left(\alpha_{j}, \beta_{j}\right)\right]$ consisting of all $V(z) \in S^{\circ 1}(R)$ such that $V(z)$ is holomorphic and positive (negative) on all intervals $\left(\alpha_{j}, \beta_{j}\right)$.

Let $\theta$ be a conservative scattering system of the form (7) such that $V_{\Theta}(z)=V(z)$ and let $A$ and $T$ be the operators of (6). Then (cf. [5])

$$
\begin{array}{ll}
\overline{\mathfrak{D}(A)}=\mathfrak{S}, & \mathfrak{D}(T) \neq \mathfrak{D}\left(T^{*}\right) \text { if } V(z) \in S_{+}^{o}\left[R, \bigcup_{j=1}^{m}\left(\alpha_{j}, \beta_{j}\right)\right], \\
\overline{\mathfrak{D}(A)} \neq \mathfrak{S}, & \mathfrak{D}(T)=\mathfrak{D}\left(T^{*}\right) \text { if } V(z) \in S_{ \pm}^{\mathfrak{1}}\left[R, \bigcup_{j=1}^{m}\left(\alpha_{j}, \beta_{j}\right)\right], \\
\overline{\mathfrak{D}(A)} \neq \mathfrak{S}, & \mathfrak{D}(T) \neq \mathfrak{D}\left(T^{*}\right) \text { if } V(z) \in S_{ \pm}^{\mathcal{O}}\left[R, \bigcup_{j=1}^{m}\left(\alpha_{j}, \beta_{j}\right)\right] .
\end{array}
$$

Theorem 7: A function $V(z)$, whose values are operators in a finite-dimensional Hilbert space $E$, belongs to the class $S_{ \pm}^{\circ}[R,(\alpha, \beta)]$ if and only if the following two conditions hold:

(i) $V(z) \in S^{\circ}(R)$.

(ii) $\frac{\beta-z}{\alpha-z} V(z) \in S^{\circ}(R)\left(\frac{\alpha-z}{\beta-z} V(z) \in S^{\circ}(R)\right.$, respectively $)$.

Proof: Assume that the conditions (28) hold. Since $S^{\circ}(R) \in S$, we have $V(z) \in S_{\downarrow}[R,(\alpha, \beta)]$ by Theorem 2 and, hence, $V(z) \in S_{ \pm}^{\circ}[R,(\alpha, \beta)]$ because $V(z) \in S^{\circ}(R)$. Conversely, assume that $V(z) \in S_{+}^{\circ}[R,(\alpha, \beta)]$. Then, clearly, $V(z) \in S^{\alpha}(R)$. It remains to show the first inclusion of (ii). It is well known that

$$
\int_{0}^{\infty}(d \sigma(t) f, f)=\lim _{\eta \uparrow \infty}(\eta \operatorname{Im} V(i \eta) f, f),
$$

where $\sigma(t)$ is the operator-valued measure of the representation (23) (cf. [6]). The definitive of $S^{\circ}(R)$ and (29) imply

$$
\lim _{\eta \uparrow \infty}(\eta \operatorname{Im} V(i \eta) f, f)=\infty \text {. }
$$

Since $V(z)=K^{\bullet}\left(\left.A\right|_{R}-z I\right)^{-1} K$, we obtain

$$
\operatorname{Im} V(z)=\operatorname{Im} z K^{\bullet}\left(\left.A\right|_{R}-\bar{z} J\right)^{-1}\left(\left.A\right|_{R}-z I\right)^{-1} K
$$

Set

$$
f_{\eta}=\left(\left.A\right|_{R}-\text { in } l\right)^{-1} K f .
$$

From (30) - (32) it follows

$$
\lim _{\eta \uparrow \infty} \eta^{2}\left(f_{\eta}, f_{\eta}\right)=\lim _{\eta \uparrow \infty} \eta^{2}\left(\left(\left.A\right|_{R}-i \eta I\right)^{-1} K f,\left(\left.A\right|_{R}-i \eta I\right)^{-1} K f\right)
$$




$$
=\lim _{\eta \uparrow \infty} \eta^{2}\left(K^{\bullet}\left(\left.A\right|_{R}+i \eta I\right)^{-1}\left(\left.A\right|_{R}-i \eta I\right)^{-1} K f, f\right)=\lim _{\eta \uparrow \infty}(\eta \operatorname{lm} V(i \eta) f, f)=\infty .
$$

We will show that

$$
\lim _{\eta \uparrow \infty}\left(\eta \operatorname{Im} \frac{\beta-i \eta}{\alpha-i \eta} V(i \eta) f, f\right)=\infty .
$$

In fact, setting $z_{i}=z_{l}=$ in in the inequality (18) and regarding the considerations of the proof of this inequality we obtain

$$
\begin{aligned}
\lim _{\eta \uparrow \infty}\left(\eta \operatorname{Im} \frac{\beta-i \eta}{\alpha-i \eta} V(i \eta) f, f\right) & =\lim _{\eta \uparrow \infty}\left(\frac{\eta^{2}(\beta-\alpha)}{\alpha^{2}+\eta^{2}}\left(\left.A\right|_{R} f_{\eta}, f_{\eta}\right)+\frac{\eta^{2} \alpha \beta}{\alpha^{2}+\eta^{2}}\left(f_{\eta}, f_{\eta}\right)+\frac{\eta^{4}}{\alpha^{2}+\eta^{2}}\left(f_{\eta}, f_{\eta}\right)\right) \\
& \geq \lim _{\eta \uparrow \infty} \frac{\eta^{4}}{\alpha^{2}+\eta^{2}}\left(f_{\eta}, f_{\eta}\right)=\lim _{\eta \uparrow \infty} \frac{\eta^{2}}{\alpha^{2}+\eta^{2}} \eta^{2}\left(f_{\eta}, f_{\eta}\right)=\infty .
\end{aligned}
$$

We mention that we have also used Theorem $4\left(\left.A\right|_{R} \geq 0\right)$ and (33).

Finally, assume that $V(z) \in S_{-}^{\circ}[R,(\alpha, \beta)]$. We will show that $((\alpha-z) /(\beta-z)) V(z) \in S^{\circ}(R)$. Setting $w=(\alpha-i \eta)(\beta-i \eta)$ and using (13) we get

$$
\begin{aligned}
(\operatorname{Im} w V(\mathrm{i} \eta) f, f) & =\frac{1}{2}\left(\left(w K^{*}\left(\left.A\right|_{R}-\mathrm{i} \eta I\right)^{-1} K-\bar{w} K^{*}\left(\left.A\right|_{R}+\mathrm{i} \eta I\right)^{-1} K\right) f, f\right) \\
& =\frac{1}{2 \mathrm{i}}\left(\left(w\left(\left.A\right|_{R}-\mathrm{i} \eta I\right)^{-1}-\bar{w}\left(\left.A\right|_{R}+\mathrm{i} \eta I\right)^{-1}\right) K f, K f\right) \\
& =\left(\left.\frac{w-\bar{w}}{2 \mathrm{i}} A\right|_{R} f_{\eta}, f_{\eta}\right)+\frac{w-\bar{w}}{2} \eta\left(f_{\eta}, f_{\eta}\right) \\
& =\frac{\eta(\alpha-\beta)}{|\beta-i \eta|^{2}}\left(\left.A\right|_{R} f_{\eta}, f_{\eta}\right)+\frac{\eta\left(\alpha \beta+\eta^{2}\right)}{|\beta-i \eta|^{2}}\left(f_{\eta}, f_{\eta}\right)
\end{aligned}
$$

where $f_{\eta}$ has the form (32). But $\left(A_{R} f_{\eta}, f_{\eta}\right)=\left(\frac{\operatorname{Imin} V(i \eta)}{\eta} f, f\right)$. In fact,

$$
\begin{aligned}
\left(\frac{\operatorname{Imi\eta } V(i \eta)}{\eta} f, f\right) & =\left(\frac{i \eta V(i \eta)+i \eta V(-i \eta)}{2 i \eta} f, f\right) \\
& =\left(\frac{i \eta K^{*}\left(\left.A\right|_{R}-i \eta I\right)^{-1} K+i \eta K^{*}\left(\left.A\right|_{R}+i \eta I\right)^{-1} K}{2 i \eta} f, f\right) \\
& =\left(\frac{\left(\left.A\right|_{R}+i \eta I\right)^{-1}\left(i \eta\left(\left.A\right|_{R}+i \eta I\right)+i \eta\left(\left.A\right|_{R}-i \eta I\right)\right)\left(A A_{R}-i \eta I\right)^{-1}}{2 i \eta} K f, K f\right) \\
& =\left(A l_{R} f_{\eta}, f_{\eta}\right) .
\end{aligned}
$$

Regarding (23) we obtain

$$
\left(\frac{\operatorname{Imin} V(\mathrm{in})}{\eta} f, f\right)=(\gamma f, f)+\int_{0}^{\infty} \frac{t}{t^{2}+\eta^{2}}(d \sigma(t) f, f) .
$$

Using Lebesgue's Dominated Convergence Theorem (cf. [6]), it follows

$$
\left.\lim _{\eta \uparrow \infty}(A\rfloor_{R} f_{\eta}, f_{\eta}\right)=\lim _{\eta \uparrow \infty}\left(\frac{\operatorname{Imin} V(\mathrm{i} \eta)}{\eta} f, f\right)=(\gamma f, f)<\infty .
$$

Now (35) and (36) imply

$$
\lim _{\eta \uparrow \infty}\left(\eta \operatorname{Im} \frac{\alpha-i \eta}{\beta-i \eta} V(i \eta) f, f\right)=\lim _{\eta \uparrow \infty}\left(\frac{\eta^{2}(\alpha-\beta)}{\beta^{2}+\eta^{2}}\left(\left.A\right|_{R} f_{\eta}, f_{\eta}\right)+\frac{\eta^{2} \alpha \beta}{\beta^{2}+\eta^{2}}\left(f_{\eta}, f_{\eta}\right)+\frac{\eta^{2}}{\beta^{2}+\eta^{2}} \eta^{2}\left(f_{\eta}, f_{\eta}\right)\right)
$$




$$
2 \lim _{\eta \uparrow \infty}\left(\frac{\eta^{2}(\alpha-\beta)}{\beta^{2}+\eta^{2}}\left(\left.A\right|_{R} f_{\eta}, f_{\eta}\right)+\frac{\eta^{2}}{\beta^{2}+\eta^{2}} \eta^{2}\left(f_{\eta}, f_{\eta}\right)\right) \geq \infty .
$$

Thus the theorem is proved

Theorem 8: A function $V(z)$, whose values are operators in a finite-dimensional Hilbert space $E$, belongs to the class $S_{ \pm}^{1}[R,(\alpha, \beta)]$ if and only if the following two conditions hold:

(i) $V(z) \in S^{4}(R)$.

(ii) $\frac{\beta-z}{\alpha-z} V(z) \in S^{\mathcal{L}}(R)\left(\frac{\alpha-z}{\beta-z} V(z) \in S^{\mathcal{L}}(R)\right.$, respectively).

Proof: The sufficiency is obvious (compare the proof of Theorem 7$)$. Now assume that $V(z)$ $\in S_{+}^{1}[R,(\alpha, \beta)]$. Clearly, $V(z) \in S^{1}(R)$. We will show that $((\beta-z) /(\alpha-z)) V(z) \in S^{1}(R)$. Since $V(z)$ is realizable, the relation (13) holds. In this relation, the operator $A_{R}$ is a bounded linear operator from $\mathfrak{W}_{\text {+ }}$ into $\mathfrak{W}_{-}$. Let $R$ be the (isometric) Riesz-Berezanskii operator, which arises in a natural way in the therory of nested Hilbert spaces (cf. [2]). The operator $R$ has the properties $(f, g)_{-}=(R f, R g)_{+}=(R f, g)=(f, R g)\left(f, g \in \mathfrak{S}_{-}\right)$. Thus

$$
\begin{aligned}
\left|\left(\left.A\right|_{R} f_{\eta}, f_{\eta}\right)\right| & =\left\|( \mathbb { R } A \| _ { R } f _ { \eta } , f _ { \eta } ) _ { + } \left|\leq\left\|\left.\mathbb{R} A\right|_{R}\right\|\left\|f_{\eta}\right\|_{+}^{2}=\left\|\left.\mathbf{R} A\right|_{R}\right\|\left(\left\|f_{\eta}\right\|^{2}+\left\|A^{*} f_{\eta}\right\|^{2}\right)\right.\right. \\
& =\left\|\left.\mathbb{R} A\right|_{R}\right\|\left(\left\|f_{\eta}\right\|^{2}+\eta^{2}\left\|P f_{\eta}\right\|^{2}\right) \leq\|\mathbf{R} A\|_{R}\left\|\left(1+\eta^{2}\right)\right\| f_{\eta} \|^{2},
\end{aligned}
$$

where $P$ is the orthoprojector of $\mathfrak{S}$ onto $\overline{\mathfrak{D}(\bar{A})}$ and the operator $A$ is the maximal common Hermitian part of the operators $T$ and $T^{*}$ that arise realizing the operator-valued function $V(z)$ as a transfer function of the conservative scattering system (7). Furthermore, as it was noted in the proof of Theorem 7, we have

$$
\lim _{\eta \uparrow \infty}\left(\eta \operatorname{Im} \frac{\beta-i \eta}{\alpha-i \eta} V(i \eta) f, f\right)=\lim _{\eta \uparrow \infty}\left(\frac{\eta^{2}(\beta-\alpha)}{\alpha^{2}+\eta^{2}}\left(\left.A\right|_{R} f_{\eta}, f_{\eta}\right)+\frac{\eta^{2} \alpha \beta}{\alpha^{2}+\eta^{2}}\left(f_{\eta}, f_{\eta}\right)+\frac{\eta^{\star}}{\alpha^{2}+\eta^{2}}\left(f_{\eta}, f_{\eta}\right)\right)
$$

Since $\int_{0}^{\infty}(d \sigma(t) f, f)=\lim _{\eta \uparrow \infty} \eta^{2}\left(f_{\eta}, f_{\eta}\right)<\infty$, the realization (38) implies $\lim _{\eta \uparrow \infty}\left(\eta ! m_{\alpha}^{\beta} \frac{-i \eta}{-i \eta} V(i \eta) f, f\right)$ $<\infty$. Now assume $V(z) \in S_{-}^{1}[R,(\alpha, \beta)]$. We will show that $((\alpha-z) /(\beta-z)) V(z) \in S^{1}(R)$. Using (37) we get

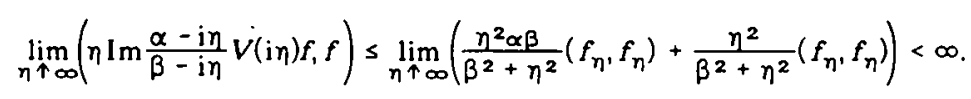

In order to obtain the last estimates we have used the fact that $\left.A\right|_{R} \geq 0$

The following theorem is an immediate consequence of Theorems 7 and (8).

Theorem 9: A function $V(z)$, whose values are operators in a finite-dimensional Hilbert space $E$, belongs to the class $S_{ \pm}^{01}[R,(\alpha, \beta)]$ if and only if the following two conditions hold:

(i) $V(z) \underline{\epsilon} S^{\circ \mathcal{L}}(R)$.

(ii) $\frac{\beta-z}{\alpha-z} V(z) \in S^{\circ y}(R)\left(\frac{\alpha-z}{\beta-z} V(z) \in S^{\circ 1}(R)\right.$, respectively).

Combining the results of Theorems $7-9$ and regarding (27) we obtain the following 
Theorem 10: A function $V(z)$, whose values are operators in a finite-dimensional Hilbert space $E$, belongs to the class $S_{ \pm}[R,(\alpha, \beta)]$ if and only if the following two conditions hold:

(i) $V(z) \in S(R)$.

(ii) $\frac{\beta-z}{\alpha-z} V(z) \in S(R) \quad\left(\frac{\alpha-z}{\beta-z} V(z) \in S(R)\right.$, respectively $)$.

Theorem 11: A function $V(z)$, whose values are operators in a finite-dimensional Hilbert space $E$, belongs to the class $S_{+}^{\circ}\left[R, \bigcup_{j=1}^{m}\left(\alpha_{j}, \beta_{j}\right)\right]$ if and only if the following two conditions hold:

(i) $V(z) \in S^{\circ}(R)$.

(ii) $\prod_{j=1}^{m} \frac{\beta_{j}-z}{\alpha_{j}-z} V(z) \in S^{\circ}(R)\left(\prod_{j=1}^{m} \frac{\alpha_{j}-z}{\beta_{j}-z} V(z) \in S^{\circ}(R)\right.$, respectively).

Proof: The sufficiency of the conditions is easy to prove. Since $S^{\circ}(R) \subset S$, we have $V(z)_{\epsilon}$ $S_{ \pm}\left[\bigcup_{j=1}^{m}\left(\alpha_{j}, \beta_{j}\right)\right]$ because of Theorem 2. But since $V(z) \in S^{\circ}(R)$, we obtain $V(z) \in S_{ \pm}^{o}\left[R, \bigcup_{j=1}^{m}\left(\alpha_{j}, \beta_{j}\right)\right]$.

The necessity is proved with aid of mathematical induction. For $n=1$ the result was proved in Theorem 7. Now assume that for $m=p$ from $V(z) \in S_{+}^{\circ}\left[R, \bigcup_{j=1}^{p}\left(\alpha_{j}, \beta_{j}\right)\right]$ it follows that

(i) $V(z) \in S^{\circ}(R)$.

(ii) $\prod_{j=1}^{p} \frac{\beta_{j}-z}{\alpha_{j}-z} V(z) \in S^{\circ}(R)$.

We will show that this fact remains true for $n=p+1$. Assume that $V(z) \in S_{+}^{\circ}\left[R, \bigcup_{j=1}^{p+1}\left(\alpha_{j}, \beta_{j}\right)\right]$. Then, clearly, $V(z) \in S_{+}^{\circ}\left[R, \bigcup_{j=1}^{p}\left(\alpha_{j}, \beta_{j}\right)\right]$ and hence

(i) $V(z) \in S^{\circ}(R)$.

(ii) $\prod_{j=1}^{p+1} \frac{\beta_{j}-z}{\alpha_{j}-z} V(z) \in S^{\circ}(R)$.

Since $V(z)$ is holomorphic and positive on the interval $\left(\alpha_{p+1}, \beta_{p+1}\right)$, we obtain

$$
\prod_{j=1}^{p} \frac{\beta_{j}-z}{\alpha_{j}-z} V(z) \in S_{+}^{\circ}\left[R,\left(\alpha_{p+1}, \beta_{p+1}\right)\right]
$$

Hence by Theorem 7,

$$
\frac{\beta_{p+1}-z}{\alpha_{p+1}-z} \prod_{j=1}^{p} \frac{\beta_{j}-z}{\alpha_{j}-z} V(z)=\prod_{j=1}^{p+1} \frac{\beta_{j}-z}{\alpha_{j}-z} V(z) \in S^{\circ}(R) .
$$

An analogous proof works in the case $V(z) \in S_{-}^{o}\left[R, \bigcup_{j=1}^{n}\left(\alpha_{j}, \beta_{j}\right)\right]$

It is not hard to see that for the classes

$$
S_{ \pm}^{1}\left[R, \bigcup_{j=1}^{m}\left(\alpha_{j}, \beta_{j}\right)\right], S_{ \pm}^{01}\left[R, \bigcup_{j=1}^{m}\left(\alpha_{j}, \beta_{j}\right)\right] \text { and } S_{ \pm}\left[R, \bigcup_{j=1}^{m}\left(\alpha_{j}, \beta_{j}\right)\right]
$$

analogous results hold. Combining the above stated theorems we get the following

Theorem 12: A function $V(z)$, whose values are operators in a finite-dimensional Hilbert space $E$, belongs to the class $S_{+}\left[R, \cup_{j=1}^{m}\left(\alpha_{j}, \beta_{j}\right)\right] \cap S_{-}\left[\cup_{k=1}^{n}\left(c_{k}, d_{k}\right)\right]$ if and only if the following two conditions hold:

(i) $V(z) \in S(R)$. 
(ii) $\prod_{j=1}^{m} \frac{\beta_{j}-z}{\alpha_{j}-z} \prod_{k=1}^{n} \frac{c_{k}-z}{d_{k}-z} V(z) \in S(R)$

Note that analogous results can be formulated for the classes

$$
\begin{aligned}
& S_{+}^{0}\left[R, \bigcup_{j=1}^{m}\left(\alpha_{j}, \beta_{j}\right)\right] \cap S_{-}^{0}\left[\bigcup_{k=1}^{m}\left(c_{k}, d_{k}\right)\right], \\
& S_{+}^{1}\left[R, \bigcup_{j=1}^{m}\left(\alpha_{j}, \beta_{j}\right)\right] \cap S_{-}^{1}\left[\bigcup_{k=1}^{m}\left(c_{k}, d_{k}\right)\right], \\
& S_{+}^{\mathrm{O}_{1}}\left[R, \bigcup_{j=1}^{m}\left(\alpha_{j}, \beta_{j}\right)\right] \cap S_{-}^{\mathrm{O}}\left[\bigcup_{k=1}^{m}\left(c_{k}, d_{k}\right)\right] .
\end{aligned}
$$

Definition: Let $A$ be a symmetric operator in a Hilbert space $\$$. The interval $(\alpha, \beta)$ is called a gap of the operator $A$ if

$$
\left\|A f-\frac{\alpha+\beta}{2} f\right\| \geq \frac{\beta-\alpha}{2}\|f\| \text { for all } f \in \mathfrak{D}(A)
$$

Theorem 13 : Let $V(z)$ b a realizable operator-valued function in a finite-dimensional Hilbert space $E$, i.e., $V(z)=K^{*}\left(\left.A\right|_{R}-z I\right)^{-1} K$, where (6) holds. Let $\overline{\mathfrak{D}(A)}=\$$ g and $A \geq 0$. Let $(\alpha, \beta)$ be an arbitrary interval of the positive semi-axis. Then $V(z) \in S_{+}[(\alpha, \beta)]$ and $(\alpha, \beta)$ is a gap of the operator $A$ if and only if the following two conditions hold:

(i) $A I_{R} \geq 0$.

(ii) $\left(\left.A\right|_{R} \varphi, \varphi\right)+\frac{\alpha \beta}{\beta-\alpha}(\varphi, \varphi)-\frac{\beta}{\beta-\alpha}\left(A^{*} \varphi, \varphi\right)-\frac{\beta}{\beta-\alpha}\left(\varphi, A^{*} \varphi\right)+\frac{1}{\beta-\alpha}\left(A^{*} \varphi, A^{*} \varphi\right) \geq 0 \forall \varphi \in \mathfrak{J}_{+}$

Proof: Assume that (40) holds. Let $\left\{z_{i}\right\}_{i=1}^{P}$ be an arbitrary set of non-real complex numbers such that $z_{i} \neq z_{1}$. Let $N_{z_{k}}$ be the deficiency space of the operator $A$ and $\varphi_{j} \in N_{z_{j}}$. Set $\varphi$ $=\sum_{i=1}^{P}\left(\alpha-z_{i}\right)^{-1} \varphi_{i}$. Since $A^{*} \varphi_{i}=z_{i} \varphi_{i}$, we obtain from (40)

$$
\begin{aligned}
& \left(A_{R} \varphi, \varphi\right)+\frac{\alpha \beta}{\beta-\alpha}(\varphi, \varphi)-\frac{\beta}{\beta-\alpha}\left(A^{*} \varphi, \varphi\right)-\frac{\beta}{\beta-\alpha}\left(\varphi, A^{*} \varphi\right)+\frac{1}{\beta-\alpha}\left(A^{*} \varphi, A^{*} \varphi\right) \\
& =\sum_{i, l=1}^{p} \frac{1}{\left(\alpha-z_{i}\right)\left(\alpha-\overline{z_{l}}\right)}\left(A \|_{R} \varphi_{i}, \varphi_{1}\right)+\frac{\alpha \beta}{\beta-\alpha} \sum_{i, l=1}^{p} \frac{1}{\left(\alpha-z_{i}\right)\left(\alpha-\bar{z}_{l}\right)}\left\{\varphi_{i}, \varphi_{1}\right) \\
& -\frac{\beta}{\beta-\alpha} \sum_{i=1=1}^{p} \frac{1}{\left(\alpha-z_{j}\right)\left(\alpha-\bar{z}_{l}\right)}\left(A^{*} \varphi_{i}, \varphi_{l}\right)-\frac{\beta}{\beta-\alpha} \sum_{i, l=1}^{p} \frac{1}{\left(\alpha-z_{i}\right)\left(\alpha-\bar{z}_{l}\right)}\left(\varphi_{i}, A^{*} \varphi_{l}\right) \\
& +\frac{1}{\beta-\alpha} \sum_{i=1}^{p} \frac{1}{\left(\alpha-z_{i}\right)\left(\alpha-\bar{z}_{l}\right)}\left(A^{*} \varphi_{i}, A^{*} \varphi_{I}\right) \\
& =\frac{1}{\beta-\alpha} \sum_{i, l=1}^{p}\left(\left(\left.\frac{\beta-\alpha}{\left(\alpha-z_{i}\right)\left(\alpha-\overline{z_{l}}\right)} A\right|_{R}+\frac{\alpha \beta-\beta\left(z_{i}+\overline{z_{l}}\right)+z_{i} \bar{z}_{l}}{\left(\alpha-z_{i}\right)\left(\alpha-\overline{z_{l}}\right)} J\right) \varphi_{i}, \varphi_{1}\right) \\
& =\frac{1}{\beta-\alpha} \sum_{i, 1=1}^{P}\left(B\left(z_{i}, z_{1}\right) \varphi_{j}, \varphi_{1}\right) \geq 0,
\end{aligned}
$$

where $B(\lambda, \mu)$ has the form (16). Thus Theorem 4 yields $V(z) \in S_{+}[(\alpha, \beta)]$.

Now we will show that $(\alpha, \beta)$ is a gap of $A$. In fact, if the vector $\varphi$ of the inequality (40) belongs to $\mathfrak{D}(A)$, then with regard to the inclusions $A^{*} \supset A$ and $\left.A\right|_{R} \supset A$ we obrain

$$
\left(A_{R} \varphi, \varphi\right)+\frac{\alpha \beta}{\beta-\alpha}(\varphi, \varphi)-\frac{\beta}{\beta-\alpha}\left(A^{*} \varphi, \varphi\right)-\frac{\beta}{\beta-\alpha}\left(\varphi, A^{*} \varphi\right)+\frac{1}{\beta-\alpha}\left(A^{*} \varphi, A^{*} \varphi\right)
$$




$$
\begin{aligned}
& =(A \varphi, \varphi)+\frac{\alpha \beta}{\beta-\alpha}(\varphi, \varphi)+\frac{2 \beta}{\beta-\alpha}(A \varphi, \varphi)+\frac{1}{\beta-\alpha}(A \varphi, A \varphi) \\
& =\frac{\alpha \beta}{\beta-\alpha}(\varphi, \varphi)+\frac{1}{\beta-\alpha}(A \varphi, A \varphi)-\frac{\alpha+\beta}{\beta-\alpha}(A \varphi, \varphi) \geq 0 .
\end{aligned}
$$

This implies

$$
(\alpha+\beta)(A \varphi, \varphi) \leq \alpha \beta(\varphi, \varphi)+(A \varphi, A \varphi) .
$$

But conditions (39) and (41) are equivalent. In fact, if (39) holds, we get

$$
\left(\left(A-\frac{\alpha+\beta}{2} I\right) \varphi,\left(A-\frac{\alpha+\beta}{2} I\right) \varphi\right) \geq\left(\frac{\beta-\alpha}{2}\right)^{2}(\varphi, \varphi) \text {, }
$$

hence

$$
(A \varphi, A \varphi)-(\alpha+\beta)(A \varphi, \varphi)+\left(\frac{\alpha+\beta}{2}\right)^{2}(\varphi, \varphi) \geq\left(\frac{\beta-\alpha}{2}\right)^{2}(\varphi, \varphi)
$$

and $(\alpha+\beta)(A \varphi, \varphi) \leq \alpha \beta(\varphi, \varphi)+(A \varphi, \varphi)$, i.e., (41). It is not hard to see that the converse conclusion is also true. Thus, the interval $(\alpha, \beta)$ is a gap of the operator $A$.

Now let $V(z) \in S_{+}[(\alpha, \beta)]$ and the interval $(\alpha, \beta)$ be a gap of the operator $A$. Then by Theorem 4 it holds (15). As it was proved above, this yields the inequality (40) for all vectors $\varphi$ of the form

$$
\varphi=\sum_{j=1}^{p}\left(\alpha-z_{i}\right)^{-1} \varphi_{i},
$$

where $\left\{z_{j}\right\}_{j=1}^{P}$ be an arbitrary set of non-real complex numbers such that $z_{j} \neq z_{1}$ and $\varphi_{i}$ is an arbitrary vector of the deficiency space $N_{z_{i}}$. Let $S_{1}=V_{z+\bar{z}} N_{z}$, where the closure is taken with respect to the metric of the space $\mathfrak{S}$. Then, clearly, $\mathfrak{Y}=\mathfrak{S}_{2} \oplus \mathfrak{S}_{2}$, where the subspaces $\mathfrak{S}_{1}$ and $\mathfrak{S}_{2}$ are invariant subspaces of the operator $A$ and the operator $A_{2}=\left.A\right|_{\mathfrak{S}_{2}}$ is selfadjoint. Thus, $A=A_{1} \Phi A_{2}$, where $A_{1}=\left.A\right|_{\mathcal{S}_{1}}$. It is easy to see that $A^{*}=A_{1}^{*} \Phi A_{2}$. It follows that each vector $\varphi \in \mathcal{S}_{+}$can be representeds in the form $\varphi=\varphi_{1}+\varphi_{2}$, where $\varphi_{1} \in \overline{\mathfrak{D}\left(A_{1}^{*}\right)}$ and $\varphi_{2} \in \mathfrak{D}\left(A_{2}\right)$. Since the operators $A_{R}$ and $A^{*}$ are continuous operators from $\mathfrak{S}_{+}$into $\mathfrak{S}_{-}$, we can extend the inequality (40) from all vecotrs of the form (42) to all vectors $\varphi \in \overline{\mathfrak{D}\left(A_{1}^{-}\right)}$. It is easy to see that

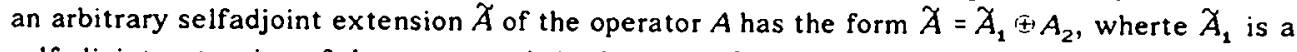
selfadjoint extension of the operator $A_{1}$ in the space $\mathfrak{S}_{1}$. Since by assumption the interval $(\alpha, \beta)$ is a gap of the operator $A$, it is also a gap of the operator $A_{2}$. Thus, for the operator $A_{2}$ it holds (39) and hence (41), as it was shown above. Setting $\varphi=\varphi_{1}+\varphi_{2}$ in (40) we obtain

$$
\begin{aligned}
\left(A_{R} \varphi, \varphi\right)+\frac{\alpha \beta}{\beta-\alpha}(\varphi, \varphi)-\frac{\beta}{\beta-\alpha}\left(A^{*} \varphi, \varphi\right)-\frac{\beta}{\beta-\alpha}\left(\varphi, A^{*} \varphi\right)+\frac{1}{\beta-\alpha}\left(A^{*} \varphi, A^{*} \varphi\right) \\
=\left(\left.A\right|_{R} \varphi_{1}, \varphi_{1}\right)+\frac{\alpha \beta}{\beta-\alpha}\left(\varphi_{1}, \varphi_{1}\right)-\frac{\beta}{\beta-\alpha}\left(A^{*} \varphi_{1}, \varphi_{1}\right)-\frac{\beta}{\beta-\alpha}\left(\varphi_{1}, A^{*} \varphi_{1}\right)+\frac{1}{\beta-\alpha}\left(A^{*} \varphi_{1}, A^{*} \varphi_{1}\right) \\
\quad+\left(\left.A\right|_{R} \varphi_{2}, \varphi_{2}\right)+\frac{\alpha \beta}{\beta-\alpha}\left(\varphi_{2}, \varphi_{2}\right)-\frac{\beta}{\beta-\alpha}\left(A^{*} \varphi_{2}, \varphi_{2}\right)-\frac{\beta}{\beta-\alpha}\left(\varphi_{2}, A^{*} \varphi_{2}\right)+\frac{1}{\beta-\alpha}\left(A^{*} \varphi_{2}, A^{*} \varphi_{2}\right) \\
=\left(\left(\left.A\right|_{R} \varphi_{1}, \varphi_{1}\right)+\frac{\alpha \beta}{\beta-\alpha}\left(\varphi_{1}, \varphi_{1}\right)-\frac{\beta}{\beta-\alpha}\left(A_{1}^{*} \varphi_{1}, \varphi_{1}\right)-\frac{\beta}{\beta-\alpha}\left(\varphi_{1}, A_{1}^{*} \varphi_{1}\right)+\frac{1}{\beta-\alpha}\left(A_{1}^{*} \varphi_{1}, A_{1}^{*} \varphi_{1}\right)\right) \\
\quad+\left(\frac{\alpha \beta}{\beta-\alpha}\left(\varphi_{2}, \varphi_{2}\right)+\frac{1}{\beta-\alpha}\left(A_{2} \varphi_{2}, A_{2} \varphi_{2}\right)-\frac{\alpha+\beta}{\beta-\alpha}\left(A_{2} \varphi_{2}, \varphi_{2}\right)\right) \geq 0 .
\end{aligned}
$$

We note that the last inequality holds since the terms in the big brackets are non-negative 
Theorem 14: Let $V(z)$ be a realizable operator-valued function in a finite-dimensional Hilbert space $E$, i.e., $V(z)=K^{\bullet}\left(\left.A\right|_{R}-z I\right)^{-1} K$, where (6) holds. Let $\overline{\mathfrak{D}(A)}=\mathfrak{S}$ and $A \geq 0$. Let $(\alpha, \beta)$ be an arbitrary interval of the positive semi-axis. Then $V(z) \in S_{-}[(\alpha, \beta)]$ and $(\alpha, \beta)$ is a gap of the operator $A$ if and only if the following two conditions hold:

(i) $\mathrm{Al} \geq 0$.

(ii) $-\left(A_{R} \varphi, \varphi\right)+\frac{\alpha \beta}{\beta-\alpha}(\varphi, \varphi)-\frac{\alpha}{\beta-\alpha}\left(A^{*} \varphi, \varphi\right)-\frac{\alpha}{\beta-\alpha}\left(\varphi, A^{*} \varphi\right)+\frac{1}{\beta-\alpha}\left(A^{*} \varphi, A^{*} \varphi\right) \geq 0 \forall \varphi \in \mathfrak{J}_{+}$.

Proof: Assume that (43) holds. Let $\left\{z_{i}\right\}_{i=1}$ be an arbitrary set of non-real complex numbers such that $z_{j} \neq z_{l}$. Let $N_{z_{i}}$ be the feficiency space of the operator $A$ and $\varphi_{j} \in N_{z_{i}}$. Setting $\varphi=\sum_{j=1}^{P}\left(\alpha-z_{i}\right)^{-1} \varphi_{j}$ in (43), we obtain with regard to $A^{*} \varphi_{i}=z_{i} \varphi_{i}$

$$
\begin{aligned}
& -\left(A_{R} \varphi, \varphi\right)+\frac{\alpha \beta}{\beta-\alpha}(\varphi, \varphi)-\frac{\alpha}{\beta-\alpha}\left(A^{*} \varphi, \varphi\right)-\frac{\alpha}{\beta-\alpha}\left(\varphi, A^{*} \varphi\right)+\frac{1}{\beta-\alpha}\left(A^{*} \varphi, A^{*} \varphi\right) \\
& =-\sum_{i, 1=1}^{P} \frac{1}{\left(\beta-z_{i}\right)\left(\beta-\overline{z_{1}}\right)}\left(A_{R} \varphi_{i}, \varphi_{1}\right)+\frac{\alpha \beta}{\beta-\alpha} \sum_{i, 1=1}^{p} \frac{1}{\left(\beta-z_{i}\right)\left(\beta-\overline{z_{l}}\right)}\left(\varphi_{i}, \varphi_{1}\right) \\
& -\frac{\alpha}{\beta-\alpha} \sum_{i, 1=1}^{p} \frac{1}{\left(\beta-z_{j}\right)\left(\beta-\overline{z_{1}}\right)}\left(A^{*} \varphi_{i}, \varphi_{1}\right)-\frac{\alpha}{\beta-\alpha} \sum_{i, 1=1}^{p} \frac{1}{\left(\beta-z_{i}\right)\left(\beta-\bar{z}_{l}\right)}\left(\varphi_{i}, A^{*} \varphi_{1}\right) \\
& +\frac{1}{\beta-\alpha} \sum_{i, l=1}^{P} \frac{1}{\left(\beta-z_{i}\right)\left(\beta-\overline{z_{l}}\right)}\left(A^{*} \varphi_{j}, A^{*} \varphi_{l}\right) \\
& =\frac{1}{\beta-\alpha} \sum_{i, I=1}^{p}\left(\left(\frac{\alpha-\beta}{\left(\beta-z_{i}\right)\left(\beta-\bar{z}_{l}\right)} A I_{R}+\frac{\alpha \beta-\alpha\left(z_{i}+\bar{z}_{l}\right)+z_{i} \bar{z}_{1}}{\left(\beta-z_{i}\right)\left(\beta-\bar{z}_{l}\right)} I\right) \varphi_{i}, \varphi_{l}\right) \\
& =\frac{1}{\beta-\alpha} \sum_{i, l=1}^{p}\left(B\left(z_{i}, z_{1}\right) \varphi_{i}, \varphi_{l}\right) \geq 0,
\end{aligned}
$$

where $B(\lambda, \mu)$ has the form (20). This implies the inclusion $V(z) \in S_{-}[(\alpha, \beta)]$ by Theorem 5 .

We will now show that $(\alpha, \beta)$ is a gap of the operator $A$. In fact, if the vector $\varphi$ in the inequality (43) belongs to $\mathfrak{D}(A)$, then with regard to the inclusions $A^{*} \supset A$ and $\left.A\right|_{R} \supset A$ we obtain

$$
\begin{aligned}
& -\left(A_{R} \varphi, \varphi\right)+\frac{\alpha \beta}{\beta-\alpha}(\varphi, \varphi)-\frac{\alpha}{\beta-\alpha}\left(A^{*} \varphi, \varphi\right)-\frac{\alpha}{\beta-\alpha}\left(\varphi, A^{*} \varphi\right)+\frac{1}{\beta-\alpha}\left(A^{*} \varphi, A^{*} \varphi\right) \\
& =-(A \varphi, \varphi)+\frac{\alpha \beta}{\beta-\alpha}(\varphi, \varphi)-\frac{2 \alpha}{\beta-\alpha}(A \varphi, \varphi)+\frac{1}{\beta-\alpha}\left(A^{*} \varphi, A^{*} \varphi\right) \\
& \quad=\frac{\alpha \beta}{\beta-\alpha}(\varphi, \varphi)+\frac{1}{\beta-\alpha}(A \varphi, A \varphi)-\frac{\alpha+\beta}{\beta-\alpha}(A \varphi, \varphi) \geq 0 .
\end{aligned}
$$

This yields $(\alpha+\beta)(A \varphi, \varphi) \leq \alpha \beta(\varphi, \varphi)+(A \varphi, A \varphi)$. Thus, the relation (41) is true for the operator $A$. As it was shown above, this implies that the interval $(\alpha, \beta)$ is a gap of $A$. The necessity part can be proved in an analogous way as the necessity part of Theorem 13

As a corollary of Theorems 13 and 14 we obtain the following general

Theorem 15: Let $V(z)$ be a realizable operator-valued function in a finite-dimensional Hilbert space $E$, i.e., $V(z)=K^{*}\left(\left.A\right|_{R}-z l\right)^{-1} K$, where (6) holds. Let $\overline{\mathfrak{D}(A)}=\$ \mathfrak{j}$ and $A \geq 0$. Let $\left(\alpha_{j}, \beta_{j}\right)$ $(j=1, \ldots, m)$ and $\left(c_{k}, d_{k}\right)(k=1, \ldots, n)$ two arbitrary sets of mutually disjoint intervals of the po- 
sitive semi-axis. Then $V(z) \in S_{+}\left[\bigcup_{j=1}^{m}\left(\alpha_{j}, \beta_{j}\right] \cap S_{-}\left[\bigcup_{k=1}^{n}\left(c_{k}, d_{k}\right)\right]\right.$ and all intervals $\left(\alpha_{j}, \beta_{j}\right)$ and $\left(c_{k}, d_{k}\right)$ are gaps of the operator $A$ if and only if the following tree conditions hold:

(i) $A \mid \geq 0$.

(ii) $\left(A_{R} \varphi, \varphi\right)+\frac{\alpha_{j} \beta_{j}}{\beta_{j}-\alpha_{j}}(\varphi, \varphi)-\frac{\beta_{j}}{\beta_{j}-\alpha_{j}}\left(A^{*} \varphi, \varphi\right)-\frac{\beta_{j}}{\beta_{j}-\alpha_{j}}\left(\varphi, A^{*} \varphi\right)+\frac{1}{\beta_{j}-\alpha_{j}}\left(A^{*} \varphi, A^{*} \varphi\right) \geq 0$ for each $\varphi \in \mathfrak{j}_{+}$and all $j=1, \ldots, m$.

$$
\text { (iii) }-\left(A_{R} \varphi, \varphi\right)+\frac{c_{k} d_{k}}{d_{k}-c_{k}}(\varphi, \varphi)-\frac{c_{k}}{d_{k}-c_{k}}\left(A^{*} \varphi, \varphi\right)-\frac{c_{k}}{d_{k}-c_{k}}\left(\varphi, A^{*} \varphi\right)+\frac{1}{d_{k}-c_{k}}\left(A^{*} \varphi, A^{*} \varphi\right) \geq 0
$$

for each $\varphi \in \mathfrak{S}_{+}$and all $k=1, \ldots, n$.

Remark: Theorems 14 and 15 were obtained in collaboration with E. R. Tsekanovskii.

\section{REFERENCES}

[1] AROV, D. Z.: Passive linear stationary dynamic systems. Sib. Math. J. 20 (1979), 211 - 228.

[2] BEREZANSKII, YU. M.: Spaces with non-positive norm. Uspekhi Math. Nauk 18 (1963) $1,63-96$.

[3] BRODSKII, M. S.: Triangular and Jordan representations of linear operators. Moscow: Nauka 1969.

[4] DERKACH, V. A. and E. R. TSEKANOVSKII: On characteristic operator functions of accretive operator nodes. Dokl. Acad. Nauk SSSR, Ser. A 8 (1981), 16 - 20.

[5] DoVZhENKO, I. N. and E. R. TSEKANOVSKII: On Classes of Stieltjes Operator Functions and their Conservative Realizations. Dokl. Acad. Nauk SSSR 311 (1990), $17-22$.

[6] KAC, I. S. and M. G. KREIN: $R$-Functions are analytical functions mapping the upper half-plane into itself. In: Discrete and Continuous Boundary Value Problems (ed.: F. Atkinson). Moscow: Nauka 1968, pp. $629-647$.

[7] KREIN, M. G. and A. A. NUDELMAN: Markov's Problem of Moments and Extremal Problems. Moscow: Nauka 1973.

[8] KREIN, M.G.: On a generalization of Stieltjes' investigations. Dokl. Acad. Nauk SSSR 82 (1952), $881-884$.

[9] Livsic, M. S.: Operators, Oscillations, Waves. Moscov: Nauka 1966.

[10] TSEKANOVSKII, E. R.: Generalized selfadjoint extensions of symmetric operators. Dok1. Acad. Nauk SSSR 178 (1968), 1267 - 1270.

[11] TSEKANOVSKII, E. R. and YU. L. SHMULJAN: Biextension operator theory in rigged Hilbert spaces. Unbounded operator nodes and characteristic functions. Uspekhi Math. Nauk 32 (1977) 5, $69-124$.

Received 16.06.1991

Dr. Vladislavl Eduardovic Tsekanovskii

Artema $84 \mathrm{kw} .65$

340055 - Donetsk, Ukraine 